\title{
Prevalence of Addictive Behaviors in Medical Students and Their Association With Stress
}

\author{
Jimin Lee ${ }^{1,2}$, Seunghee Won ${ }^{1,2}$, Sung Man Chang ${ }^{1,2}$, Byung-Soo Kim ${ }^{1,3}$, and Seung Jae Lee ${ }^{1,2} \bowtie$ \\ ${ }^{1}$ Department of Psychiatry, School of Medicine, Kyungpook National University, Daegu, Republic of Korea \\ ${ }^{2}$ Department of Psychiatry, Kyungpook National University Hospital, Daegu, Republic of Korea \\ ${ }^{3}$ Department of Psychiatry, Kyungpook National University Chilgok Hospital, Daegu, Republic of Korea
}

\begin{abstract}
Objective This study aims to investigate the prevalence of the addictive use of the internet, smartphone, and alcohol in medical students, the association of this addictive use with stress, and the mediating roles of resilience and self-esteem in this association.

Methods A total of 866 medical students completed measures of three addictive uses as well as psychological scales for stress, resilience, and self-esteem. Correlation analyses and parallel mediation analysis were carried out.

Results The prevalence of potential-risk and high-risk users was $5.8 \%$ and $1.7 \%$ for internet use, $5.4 \%$ and $2.2 \%$ for smartphone use, $22.6 \%$ and 5.3\% for alcohol use, respectively. All three addictive behaviors tended to increase in terms of prevalence or mean score according to an increase in a students' grade. Stress was positively correlated with internet use $(r=0.324, \mathrm{p}<0.001)$ and smartphone use ( $\mathrm{r}=0.347$, $\mathrm{p}<0.001$ ). Resilience and self-esteem were found to be mediators in the association between stress and internet use or smartphone use.

Conclusion These findings suggest that addictions in medical students are as prevalent as in a general population and that internet use and smartphone use may be better explained by a stress-addiction model with resilience and self-esteem as mediators than alcohol use.
\end{abstract}

Psychiatry Investig 2022;19(1):44-53

Keywords Medical students; Stress; Addictive behavior; Psychological resilience; Self-esteem.

\section{INTRODUCTION}

Since university students are important resources who will take charge of future leading roles and positions in society, their mental health has been regarded as an important aspect in the development of individuals and society. ${ }^{1}$ Due to the current trend in which university courses tend to be considered as stepping stones to success rather than be chosen based on student's abilities and aptitudes, university students are under a lot of stress due to excessive competition and preparation for study and employment. ${ }^{2,3}$ Medical students tend to have less stress about employment or anxiety about the future than their non-medical academic peers, but their academic stress is severe due to excessive learning and relative sense of

Received: March 14, 2021 Revised: September 10, 2021

Accepted: October 27, 2021

$\triangle$ Correspondence: Seung Jae Lee, MD, PhD

Department of Psychiatry, School of Medicine, Kyungpook National University, 680 Gukchaebosang-ro, Jung-gu, Daegu 41944, Republic of Korea Tel: +82-53-200-5747, Fax: +82-53-426-5361, E-mail: jayleemd@knu.ac.kr

(a) This is an Open Access article distributed under the terms of the Creative Commons Attribution Non-Commercial License (https://creativecommons.org/licenses/bync/4.0) which permits unrestricted non-commercial use, distribution, and reproduction in any medium, provided the original work is properly cited. failure among their peers. They also have high level of anxiety due to repetitive tests, extreme competition, and flunk system. Therefore, mental problems may occur more frequently in medical students than those in the general population. ${ }^{4}$ In particular, medical students have experienced addiction as well as depression, anxiety, and suicidal ideation..$^{5-8}$ Since the mental health of medical students is associated with the quality of health care in the future, it is essential to propose an intervention strategy for the prevention and treatment of these conditions.

Previous studies have investigated the prevalence of highrisk and potential-risk users of each addictive behavior in medical students. For risky users, the prevalence ranged from $5.6 \%-52.4 \%$ for internet use, $4.4 \%-60.3 \%$ for smartphone use, and $15.4 \%-45.5 \%$ for alcohol use. ${ }^{5,10-14}$ Some prevalence values seem high relative to the prevalence of the general adult population. ${ }^{15,16}$ However, these studies most reported only one or two types of addiction and did not show overall pattern of addiction across several types of addictive behavior. Moreover, only a few studies have compared the levels of addiction among medical students by grade, mostly due to small study sample sizes. 
Stress is a known risk factor for the development of addiction. ${ }^{17}$ From a biological perspective, stress increases an individual's vulnerability to addiction through mechanisms, such as chronic stress-mediated changes in the dopaminergic system. ${ }^{17}$ Also, stress affects the catecholaminergic modulation of prefrontal circuits, which in turn impairs executive functions such as inhibitory processes, which are associated with selfcontrol and impulse control. ${ }^{18}$ From a psychological perspective, impaired coping skills and an increased sensitivity to negative emotions also increase an individual's vulnerability to addiction. ${ }^{17,18}$

As such, many studies have compared the association between addictive behavior and stress, but only a few studies have been conducted on medical students. Among them, studies on internet use and smartphone use have shown a positive correlation between stress and addictive behaviors, but studies on alcohol use are not clear, as they show either negative correlation or no correlation. ${ }^{5-8,19,20}$ Although more studies are needed, these mixed findings may indicate that many internal and external factors are involved in the relationship between stress and addiction. The current study focused on the protective psychological factors, specifically psychological resilience and self-esteem that can reduce addictive behavior.

Psychological resilience refers to effective coping and adaptation to adverse situations in a positive manner. ${ }^{21,22}$ Resilient individuals use positive emotions to alleviate the effects of stress and show physiological differences in their capability to adapt to stress. ${ }^{21}$ It was also found that the higher the level of a participant's resilience, the lower the level of internet addiction. ${ }^{22}$ On the other hand, self-esteem is defined as a global barometer of self-evaluation that involves a cognitive appraisal about general self-worth and affective experiences of the self. ${ }^{23}$ A high level of self-esteem equips individuals with the abilities to handle unpleasant situations, cope effectively with challenges, and positively mediate the expression of dysfunctional schema when negative life events are experienced. Previous studies found a significant correlation between selfesteem and internet addiction in university students as well as a mediating effect of self-esteem between stress and substance abuse among adolescents. ${ }^{24,25}$ To the best of our knowledge, despite the obvious relationships between addictive behaviors, stress, and resilience or self-esteem, no studies have been conducted on the mediating roles of resilience and self-esteem in the relationship between stress and various addictive behaviors among medical students.

Therefore, the aim of this study is to simultaneously investigate the levels of internet addiction, smartphone addiction, and alcohol addiction in a relatively large number of medical students and to determine differences according to grades. In addition, this study aims to investigate the relationship be- tween stress and addictive behaviors, as well as the roles of resilience and self-esteem as mediating factors.

\section{METHODS}

\section{Research design and sample}

The Kyungpook National University School of Medicine conducted mental health assessments on students annually from 2009, and the data used in this study were drawn from three grades (premedical first grade, medical first grade, medical third grade) from 2017 to 2020 . The students responded to self-report questionnaires through a computer-based test. In the case of the results of the subjects who performed the tests more than once as follow-up observations, only the first test results were analyzed.

In proportion to the sex distribution of all medical students, the data of 866 students, consisting of 613 men (70.8\%) and 253 women $(29.2 \%)$, were included in the final analysis. According to the distribution by grade, $356(41.1 \%)$ were firstyear premedical students, 297 (34.3\%) were first-year medical students, and 213 (24.6\%) were third-year medical students.

This study was approved by the Institutional Review Board of Kyungpook National University School of Medicine (IRB No. 2021-0086). All of the subjects were fully informed about the aims and methods of the study, participated voluntarily, and gave written informed consent prior to their participation in this study.

\section{Measurement of addiction}

\section{Internet addiction}

The short form internet addiction proneness scale for adult: self-report, developed by the National Information Society Agency, was used to measure internet addiction (Cronbach's alpha $[\alpha]=0.87) .{ }^{26}$ It consists of a total of 15 questions, and each item is evaluated with a score from 1 (not at all) to 4 (extremely). The question is composed of four sub-factors: daily life impairment, virtual world orientation, withdrawal, and tolerance. In accordance with grading standards, the sample was classified into three groups according to the level of internet addiction measured: high-risk user groups, potential-risk user groups, and general user groups. ${ }^{26} \mathrm{~A}$ high-risk user group is one that has a total score of 42 or higher or that meets all 14 or more points of daily living disability, 12 or more points of withdraw$\mathrm{al}$, and 13 or more points of tolerance. A potential-risk user group has a total score of 39 to 41 points or 14 or more points of daily living disability. A general user group is a group that does not belong to either of the other two groups. 


\section{Smartphone addiction}

To measure smartphone addiction, we used the Korean smartphone addiction proneness scale for adults, which was developed by the National Information Society Agency (Cronbach's $\alpha=0.81) .{ }^{27}$ Each of the sub-factors, including daily life impairment, virtual world orientation, withdrawal, and tolerance, consists of a total 15 questions and is rated from 1 (not at all) to 4 (extremely) points. The sample was classified into three groups according to the scoring criteria of the National Information Society Agency. A high-risk user group is one that has a total score of 44 or higher or that meets all 15 or more points of daily living disability, 13 or more points of withdraw$\mathrm{al}$, and 13 or more points of tolerance. A potential-risk user group has a total score of 40 to 43 points or 14 or more points of daily living disability. In addition, a general user group is a group that does not belong to either of the other two groups.

\section{Alcohol addiction}

The Alcohol Use Disorders Identification Test (AUDIT), which was developed by the World Health Organization (WHO) to screen dangerous drinkers, was used to measure the levels of alcohol addiction among the students. The scale consists of 10 questions regarding drinking behaviors (3 questions), dependence symptoms (3 questions), and alcohol-related problems ( 4 questions) and is evaluated on a scale of 0 to 4 points. The Korean version of AUDIT is based on the amount of Korean liquor and has been validated on the Korean population (Cronbach's $\alpha=0.92) .{ }^{28}$ According to the Korean version standard, ${ }^{29}$ men who scored more than 20 points as well as women who scored more than 10 points were classified into the high-risk drinking group (the supposed alcohol use disorder group), and men who scored between 10 and 19 points as well as women who scored between 6 and 9 points were classified into the at-risk drinking group. In addition, a general user group is a group that does not belong to either of the other two groups.

\section{Measurement of stress, resilience, and self-esteem}

The Perceived Stress Scale (PSS) is an instrument used to measure the level of stress experienced over the past month. In this study, we used the Korean version of the PSS, which has a Cronbach's $\alpha$ value of $0.82 .^{30}$ A total of 10 questions are evaluated along a scale of 0 (never) to 4 (very often) points. Questions 4, 5, 7, and 8 are negative questions and are backscored. The total range is 0 to 40 points. The higher the total score, the greater the perceived level of stress.

The Connor-Davidson Resilience Scale (CD-RISC) is used to evaluate resilience related to coping skills in stressful events. The Korean version of the CD-RISC features excellent internal consistency and was developed in 2010 (Cronbach's $\alpha>0.9$ ). ${ }^{31}$
It consists of a total of 25 questions, and each item is evaluated with a score of 0 (not at all) to 4 (extremely) points. The scores of all items are summed, with a higher score indicating greater psychological resilience.

The Rosenberg Self-Esteem Scale is a measurement that evaluates an individual's self-esteem, which is the level to which one respects oneself and the level to which one considers oneself worthwhile. A total of 10 questions are evaluated with a score of 0 (not at all) to 3 (extremely) points, and Questions $3,5,8,9$, and 10 are scored inversely. Higher scores indicate higher self-esteem. In this study, the scale tool which was developed by Rosenberg and cited by Yoo et al. ${ }^{32}$ was used (Cronbach's $\alpha=0.86$ ).

\section{Statistical analysis}

For each addictive behavior (internet, smartphone, and alcohol use), we used Pearson's chi-square test to compare the sex and grade-level differences in the prevalence of high-risk and potential-risk groups. A one-way analysis of variance was used to compare the differences in the levels of the addictive behaviors and psychological variables (stress, resilience, and self-esteem) among the three groups (premedical first grade, medical first grade, medical third grade). The same differences were also tested between male and female students by means of a t-test.

To analyze the relationship between addictive behaviors and psychological variables, zero-order and partial correlation analyses with sex and grade corrections were performed. For the purpose of correcting for multiple testing, the Bonferroni correction was applied to statistical significance calculations.

Since stress, resilience, and self-esteem are overlapping and correlated concepts, various mediation models need to be considered. However, this study focused on the roles of resilience and self-esteem as mediators in the relationship between stress and addictive behaviors, based on a previously confirmed mediation model..$^{25}$ The effects of self-esteem and resilience as mediators of the association between stress and addictive behaviors were analyzed via a parallel mediation model after controlling for sex and grade. This analysis was completed with the Statistical Package for Social Sciences (SPSS) PROCESS macro, version 3.5 (model 4). The bootstrapping method was used in this analysis. The calculated confidence intervals (CIs) were used to determine whether each effect (i.e., direct, indirect) was statistically significant. For each effect, we examined the $95 \% \mathrm{CI}$ and if the value of 0 did not fall within the range of the CI for that effect, then the effect was deemed to be statistically significant.

We performed all statistical analyses using SPSS, version 20.0 (IBM Corp., Armonk, NY, USA). For these analyses, a two-sided test at the 0.05 level was used to evaluate statistical 
significance.

\section{RESULTS}

\section{Prevalence of internet, smartphone,} and alcohol addiction

Table 1 represents the prevalence of addiction in internet, smartphone, and alcohol use. Among the entire sample of students, the prevalence of potential-risk and high-risk users was $5.8 \%$, and $1.7 \%$ for internet use, and $5.4 \%$ and $2.2 \%$ for smartphone use, respectively. Moreover, for alcohol use, the prevalence was higher than that of the other addictive behaviors, with the prevalence of at-risk drinking group being $22.6 \%$ and the prevalence of the high risk-drinking group being 5.3\%.

In terms of grade, the risky internet users, who met the highrisk or potential-risk categories, increased from $4.8 \%$ to $8.4 \%$ to $10.8 \%$ as they were going up in grade $\left(\chi^{2}=7.5, p=0.023\right)$. The risky alcohol users also tended to increase from $23.9 \%$ to $28.3 \%$ to $34.3 \%\left(\chi^{2}=7.18, p=0.028\right)$. In contrast, the number of risky smartphone users showed no statistical difference according to grade. Additionally, there was no sex difference in the prevalence of risky users of the internet and smartphone, while the prevalence of risky alcohol users was higher in females than in males $\left(\chi^{2}=13.79, p<0.001\right)$.

\section{Differences between quantitative scores of addictive behaviors and psychological variables}

Table 2 shows the quantitative differences by grade and sex for each addiction behavior. For grade-level differences, the first-year premedical students showed significantly lower scores on internet and smartphone use than both the first and thirdyear medical students did. However, there was no grade-level difference in alcohol use. Regarding the difference between the sexes, males reported higher scores than females in the inter-

Table 1. Prevalence of risky internet, smartphone, and alcohol users by grade and sex among medical students (N=866)

\begin{tabular}{|c|c|c|c|c|c|}
\hline Addictive behaviors & & Prevalence (N, \%) & & $\chi^{2 *}$ & $\mathrm{p}$ \\
\hline 1. Grade & PMED1 (N=356) & MED1 (N=297) & MED3 $(\mathrm{N}=213)$ & & \\
\hline \multicolumn{6}{|l|}{ Internet use } \\
\hline Normal & $339(95.2)$ & $272(91.6)$ & $190(89.2)$ & 7.5 & 0.023 \\
\hline Potential risk group & $14(3.9)$ & $20(6.7)$ & $16(7.5)$ & & \\
\hline High-risk group & $3(0.9)$ & $5(1.7)$ & $7(3.3)$ & & \\
\hline \multicolumn{6}{|l|}{ Smartphone use } \\
\hline Normal & $336(94.4)$ & $268(90.2)$ & $196(92.0)$ & 4.0 & 0.135 \\
\hline Potential risk group & $16(4.5)$ & $22(7.4)$ & $9(4.2)$ & & \\
\hline High-risk group & $4(1.1)$ & $7(2.4 \%)$ & $8(3.8)$ & & \\
\hline \multicolumn{6}{|l|}{ Alcohol use } \\
\hline Normal & $271(76.1)$ & $213(71.7)$ & $140(65.7)$ & 7.2 & 0.028 \\
\hline Potential risk group & $78(21.9)$ & $63(21.2)$ & $55(25.8)$ & & \\
\hline High-risk group & $7(2.0)$ & $21(7.1)$ & $18(8.5)$ & & \\
\hline 2. Sex & Total $(\mathrm{N}=866)$ & Male $(\mathrm{N}=613)$ & Female $(\mathrm{N}=253)$ & & \\
\hline \multicolumn{6}{|l|}{ Internet use } \\
\hline Normal & $801(92.5)$ & $562(91.7)$ & $239(94.5)$ & 2.0 & 0.157 \\
\hline Potential-risk group & $50(5.8)$ & $38(6.2)$ & $12(4.7)$ & & \\
\hline High-risk group & $15(1.7)$ & $13(2.1)$ & $2(0.8)$ & & \\
\hline \multicolumn{6}{|l|}{ Smartphone use } \\
\hline Normal & $800(92.4)$ & $563(91.8)$ & $237(93.7)$ & 0.9 & 0.355 \\
\hline Potential-risk group & $47(5.4)$ & $36(5.9)$ & $11(4.3)$ & & \\
\hline High-risk group & $19(2.2)$ & $14(2.3)$ & $5(2.0)$ & & \\
\hline \multicolumn{6}{|l|}{ Alcohol use } \\
\hline Normal & $624(72.1)$ & $464(75.7)$ & $160(63.2)$ & 13.8 & $<0.001$ \\
\hline Potential-risk group & $196(22.6)$ & $132(21.5)$ & $64(25.3)$ & & \\
\hline High-risk group & $46(5.3)$ & $17(2.8)$ & $29(11.5)$ & & \\
\hline
\end{tabular}

*since the number of high-risk group for all addictive behaviors was too small for analysis, the prevalence of high- plus potential-risk users were compared between groups. PMED1, premedical first grade; MED1, medical first grade; MED3, medical third grade 
Table 2. Differences in quantitative scores of addictive behaviors and psychological variables by grade and sex among medical students $(\mathrm{N}=866)$

\begin{tabular}{|c|c|c|c|c|c|c|}
\hline \multirow{2}{*}{ Variables } & \multirow{2}{*}{\multicolumn{3}{|c|}{ Quantitative scores of addictive behaviors }} & \multicolumn{2}{|c|}{ Statistics } & \multirow{2}{*}{ Post-hoc } \\
\hline & & & & \multirow[t]{2}{*}{$\mathrm{F} / \mathrm{t}$} & \multirow[t]{2}{*}{$\mathrm{p}$} & \\
\hline 1. Grade & $\mathrm{PMED1}^{\mathrm{a}}(\mathrm{N}=356)$ & $\mathrm{MED1}^{\mathrm{b}}(\mathrm{N}=297)$ & $\operatorname{MED3}^{c}(\mathrm{~N}=213)$ & & & \\
\hline \multicolumn{7}{|l|}{ Addictive behaviors } \\
\hline Internet use & $23.73 \pm 6.27$ & $26.24 \pm 6.76$ & $26.53 \pm 6.55$ & 17.2 & $<0.001$ & $\mathrm{a}<\mathrm{b}, \mathrm{a}<\mathrm{c}$ \\
\hline Smartphone use & $24.98 \pm 6.71$ & $27.32 \pm 7.75$ & $26.89 \pm 7.63$ & 9.4 & $<0.001$ & $\mathrm{a}<\mathrm{b}, \mathrm{a}<\mathrm{c}$ \\
\hline Alcohol use & $6.08 \pm 4.38$ & $6.67 \pm 4.74$ & $7.05 \pm 5.72$ & 2.9 & 0.056 & \\
\hline \multicolumn{7}{|c|}{ Psychological variables } \\
\hline Stress & $8.54 \pm 5.21$ & $12.20 \pm 5.80$ & $12.08 \pm 5.57$ & 45.2 & $<0.001$ & $\mathrm{a}<\mathrm{b}, \mathrm{a}<\mathrm{c}$ \\
\hline Resilience & $74.27 \pm 14.34$ & $70.42 \pm 17.60$ & $72.77 \pm 17.09$ & 4.6 & 0.011 & $a>b$ \\
\hline Self-esteem & $23.63 \pm 4.61$ & $22.28 \pm 5.13$ & $22.78 \pm 4.91$ & 6.4 & 0.002 & $a>b$ \\
\hline 2. Sex & Total $(\mathrm{N}=866)$ & Male $(\mathrm{N}=613)$ & Female $(\mathrm{N}=253)$ & & & \\
\hline \multicolumn{7}{|l|}{ Addictive behaviors } \\
\hline Internet use & $25.28 \pm 6.63$ & $25.91 \pm 6.73$ & $23.75 \pm 6.14$ & 4.4 & $<0.001$ & - \\
\hline Smartphone use & $26.25 \pm 7.38$ & $25.97 \pm 7.47$ & $26.94 \pm 7.14$ & -1.8 & 0.077 & - \\
\hline Alcohol use & $6.52 \pm 4.87$ & $7.07 \pm 4.84$ & $5.19 \pm 4.70$ & 5.3 & $<0.001$ & - \\
\hline \multicolumn{7}{|c|}{ Psychological variables } \\
\hline Stress & $10.67 \pm 5.78$ & $10.64 \pm 5.80$ & $10.74 \pm 5.73$ & -0.2 & 0.819 & - \\
\hline Resilience & $72.58 \pm 16.27$ & $73.18 \pm 16.63$ & $71.12 \pm 15.33$ & 1.7 & 0.091 & - \\
\hline Self-esteem & $22.96 \pm 4.90$ & $23.17 \pm 4.80$ & $22.45 \pm 5.10$ & 2.0 & 0.047 & - \\
\hline
\end{tabular}

Values are mean \pm standard deviation. Statistical significance was tested by analysis of variance (for grade distribution) and t-test (for sex distribution). PMED1, premedical first grade; MED1, medical first grade; MED3, medical third grade

Table 3. Correlations between addictive behaviors and psychological variables in medical students $(\mathrm{N}=866)$

\begin{tabular}{lcccccc}
\hline & 1 & 2 & 3 & 4 & 5 & 6 \\
\hline 1. Stress & & $-0.40^{*}$ & $-0.51^{*}$ & $0.32^{*}$ & $0.35^{*}$ & 0.04 \\
2. Resilience & $-0.40^{*}$ & & $0.48^{*}$ & $-0.39^{*}$ & $-0.43^{*}$ & -0.08 \\
3. Self-esteem & $-0.51^{*}$ & $0.48^{*}$ & & $-0.36^{*}$ & $-0.31^{*}$ & -0.01 \\
4. Internet use & $0.35^{*}$ & $-0.38^{*}$ & $-0.35^{*}$ & & $0.60^{*}$ & $0.16^{*}$ \\
5. Smartphone & $0.36^{*}$ & $-0.43^{*}$ & $-0.32^{*}$ & $0.59^{*}$ & & $0.23^{*}$ \\
$\quad$ use & & & & & & \\
6. Alcohol use & 0.07 & -0.08 & -0.01 & $0.19^{*}$ & $0.22^{*}$ & \\
\hline
\end{tabular}

Bottom-left off-diagonal correlations for zero-order correlations, top-right off-diagonal correlations for partial correlations controlling for grade and sex. ${ }^{*} \mathrm{p}<0.001$ which is below Bonferroni adjusted $\mathrm{p}<0.006$.

net and alcohol use but not in the smartphone use.

Among psychological variables, perceived stress in the firstyear premedical students was significantly lower than that in observed the other two grades, whereas resilience and self-esteem in the first-year premedical students were higher than those observed in the first-year medical students. Meanwhile, self-esteem was the only variable that showed a significant sex difference, with scores being higher in males than in females.

\section{Correlations of stress, resilience, and self-esteem with addictive behaviors}

Table 3 shows the correlations between the psychological variables and the addictive behaviors. Stress was positively correlated with internet use and smartphone use (all Ps $<0.001$ ). Resilience was negatively correlated with internet use and smartphone use (all Ps $<0.001)$. Self-esteem was also negatively correlated with internet use and smartphone use (all Ps<0.001). These relationships remained significant after controlling for sex and grade. However, the level of alcohol use was not correlated with stress, resilience, or self-esteem, regardless of whether sex and grade were controlled.

\section{The mediating effect of resilience and self-esteem on the association between stress and addictive behaviors}

The regression model for each addictive behavior was found to be statistically significant (all $\mathrm{Ps}<0.001$ ). In addition, for the analysis of statistical power, the $\mathrm{R}^{2}$ values associated with the F-tests of the addictive behavior models were: in the internet use model, 0.27 (self-esteem as the outcome), 0.16 (resilience as the outcome), and 0.25 (internet use as the outcome); in the smartphone use model, 0.27 (self-esteem as the outcome), 0.16 (resilience as the outcome) and 0.24 (smartphone use as the 
outcome); and in the alcohol use model, 0.27 (self-esteem as the outcome), 0.16 (resilience as the outcome), and 0.05 (alcohol use as the outcome). According to the standard for $\mathrm{R}^{2}$ values proposed by Cohen, ${ }^{33}$ the statistical power of our study is above the medium level $\left(\mathrm{R}^{2}=0.13\right)$, with the exception of the alcohol use model.

The mediating effects of resilience and self-esteem on stress and each addiction behavior were different (Table 4). For internet use, resilience and self-esteem mediate the relationship between stress and internet use. In addition, the sum of the in- direct effects was 0.20 , which was statistically significant, as the 95\% CI (0.15-0.24) did not include 0. For smartphone use, a statistically significant indirect effect of stress on smartphone use was revealed through resilience but not through self-esteem. Moreover, the sum of the indirect effects was 0.18 , which was statistically significant, as the 95\% CI (0.13-0.24) did not include 0 . On the other hand, for alcohol use, the $95 \%$ CIs for the indirect effect contained 0 , suggesting that neither resilience nor self-esteem was a significant mediator on the effect of stress on alcohol use. Figure 1 depicts the summary of the

Table 4. Mediating effect of resilience and self-esteem on the association between stress and addictive behaviors

\begin{tabular}{|c|c|c|c|c|}
\hline \multirow{2}{*}{ Model pathway } & \multirow{2}{*}{ Standardized estimated effect } & \multicolumn{2}{|c|}{$95 \%$ confidence interval } & \multirow{2}{*}{$\mathrm{p}$} \\
\hline & & Lower $2.5 \%$ & Upper $2.5 \%$ & \\
\hline \multicolumn{5}{|l|}{ 1. Internet use } \\
\hline \multicolumn{5}{|l|}{ Indirect effect } \\
\hline Stress $\rightarrow$ Resilience $\rightarrow$ Internet use & 0.12 & 0.08 & 0.16 & \\
\hline Stress $\rightarrow$ Self-esteem $\rightarrow$ Internet use & 0.08 & 0.03 & 0.13 & \\
\hline Direct effect & 0.18 & 0.10 & 0.26 & $<0.001$ \\
\hline Total effect & 0.38 & 0.30 & 0.45 & $<0.001$ \\
\hline \multicolumn{5}{|l|}{ 2. Smartphone use } \\
\hline \multicolumn{5}{|l|}{ Indirect effect } \\
\hline Stress $\rightarrow$ Resilience $\rightarrow$ Smartphone use & 0.18 & 0.14 & 0.23 & \\
\hline Stress $\rightarrow$ Self-esteem $\rightarrow$ Smartphone use & 0 & -0.05 & 0.06 & \\
\hline Direct effect & 0.27 & 0.18 & 0.37 & $<0.001$ \\
\hline Total effect & 0.46 & 0.37 & 0.54 & $<0.001$ \\
\hline \multicolumn{5}{|l|}{ 3. Alcohol use } \\
\hline \multicolumn{5}{|l|}{ Indirect effect } \\
\hline Stress $\rightarrow$ Resilience $\rightarrow$ Alcohol use & 0.03 & -0.01 & 0.07 & \\
\hline Stress $\rightarrow$ Self-esteem $\rightarrow$ Alcohol use & -0.03 & -0.07 & 0.01 & \\
\hline Direct effect & 0.03 & -0.04 & 0.10 & 0.360 \\
\hline Total effect & 0.04 & -0.02 & 0.10 & 0.192 \\
\hline
\end{tabular}

If the confidence interval does contain zero, the indirect effect is not significant

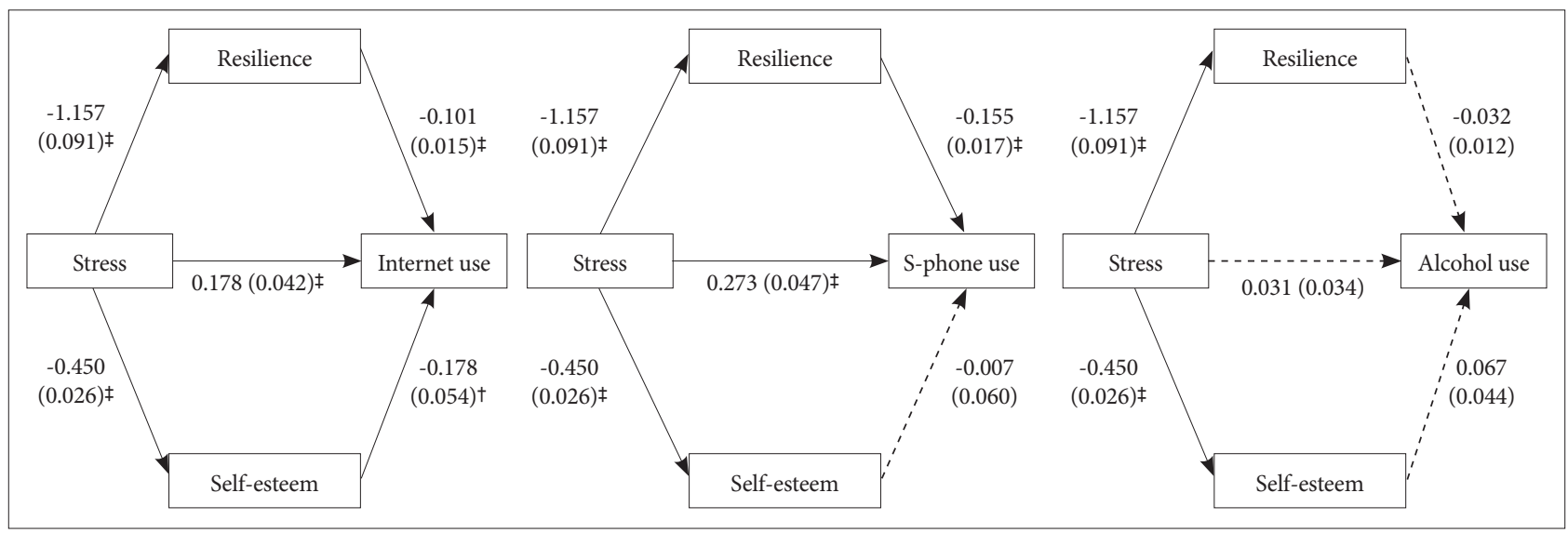

Figure 1. Mediating effects of resilience and self-esteem on the relationship between stress and internet, smartphone (S-phone), or alcohol use in medical students. Unstandardized coefficients are presented with standard errors in parentheses. ${ }^{\dagger} p<0.01 ; \neq p<0.001$. 
relationship between stress, resilience, self-esteem, and each addiction behavior.

\section{DISCUSSION}

We investigated the prevalence of three addictive behaviors and their associations with stress in medical students. The prevalence of potential-risk and high-risk users was, respectively, $5.8 \%$ and $1.7 \%$ for internet use, $5.4 \%$ and $2.2 \%$ for smartphone use, and $22.6 \%$ and $5.3 \%$ for alcohol use. All three addictive behaviors tended to increase in terms of prevalence or mean score with increasing grade levels. In addition, there were associations between internet and smartphone use and stress, in which resilience and self-esteem acted as mediators. However, the association between stress and alcohol use, and the mediating roles of them on this association were not found in alcohol use.

\section{Prevalence of risky internet, smartphone, and alcohol users}

In the literature, the prevalence of addictive behaviors of medical students varies greatly from study to study, ${ }^{5,10-14}$ For risky users, the prevalence was $5.6 \%-52.4 \%$ for internet, $4.4 \%$ $60.3 \%$ for smartphones, and $15.4 \%-45.5 \%$ for alcohol. ${ }^{5,10-14}$ These discrepancies between studies may be due to differences in the measurement scale (including cut-off values), the educational environments, the sample size and distribution, and the socio-cultural backgrounds. However, the prevalence of risky internet use and alcohol use in our study seems to be similar to that of the Korean general adult population in other surveys using the same measurement scale (internet use: $5.8 \%$, and alcohol use: $33.2 \%)^{15,16}$ Thus, the prevalence in this study is indicative of potential public health concerns regarding addictive behaviors among medical students.

\section{Grade-level differences of addictive behaviors and psychological variables}

In this study, qualitative and quantitative analyses demonstrated characteristic changes of use pattern among three grades. The mean scores of internet and smartphone use were higher in the first and third graders of the medical school than in the first graders of the premedical school. These findings indicated that the general use of the internet and smartphone was increased during this period. In contrast, an increase in the proportion of risky users was not observed in smartphone use, though it was in internet use, as grade increased.

The first graders of the premedical school who took the painful entrance exam enjoyed the joy of experienced entering medical school, so their level of stress were low, and their internet and smartphone use was believed to be less than that of other grades.$^{20}$ In fact, consistent with previous study,,$^{34}$ perceived stress was found to be higher in the first and third graders of the medical school than in the first graders of the premedical school in this study. These observations support the notion that the internet and smartphone are used as means by which to reduce stress from negative emotions. ${ }^{35}$ Another explanation of the general increase of internet and smartphone use may be due to heightened accessibility, as university students can access the internet and smartphone more freely than high school students can. Third, social influences should also be considered. Internet use and smartphone use (being connected online) have become social norms, and there are expectations of constant reachability. ${ }^{36}$ According to Erikson's developmental stages, the developmental task of early adulthood is to expand the sense of intimacy by forming meaningful interpersonal associations with others while increasing interest in others. ${ }^{37}$ In this process of psychosocial development, it is a process in which the use of the internet or smartphone itself reacts to social conformity as a result of peer pressure. ${ }^{38}$ In addition, based on the social learning theory, the use of the internet and smartphone can also be viewed as a process of adapting to social life by modeling social surroundings. ${ }^{39}$ Therefore, in the process of adapting to university life, it can be considered that the use of the internet and smartphone would gradually increase. Although both internet and smartphone use increased, the prevalence of risky users only increased with respect to internet use. We assume that risky internet users may include internet users for entertainment purpose, such as multi-user online games or gambling games, which may be more addictive than smartphone. ${ }^{40}$ Future studies are needed to see what kinds of contents lead risky internet users.

On the other hand, although no significant differences in the mean scores of alcohol use were found, risky alcohol users increased from $23.9 \%$ to $28.3 \%$ to $34.3 \%$ as grade level increased, and this prevalence was higher than that of other addictive behaviors in this study. Unlike internet and smartphone use, alcohol did not show generally increased use according to grade partially because exposure to alcohol is less frequent than internet and smartphone use. Nevertheless, the reason why the proportion of risky alcohol users increases is that alcohol addiction may be more biologically susceptible than the other addiction behaviors. Last but not least, it should be noted that there were no statistical differences between the first and third medical students in the mean scores of all addictive uses and psychological variables in this study.

\section{Sex differences of addictive behaviors}

Consistent with previous studies, ${ }^{40}$ males generally reported to use the internet more than females in this study. However, there was no difference in the prevalence of risky users. 
These results could be explained by differences in the internet usage preferences of males and females. Whereas females prefer social network services, males prefer to use the internet for entertainment purposes, such as multi-user online games, which may be more addictive. ${ }^{40}$ For smartphone use, there were no sex differences in general use or in the prevalence of risky users. Regarding alcohol use, the mean score of females was lower while the prevalence in the risk drinking group is higher in females than in males. Interpretation of these results should be cautious because the cutoff value of risky drinking is lower in for females than for males. However, this difference may not come solely from different cutoff values. In fact, the sex gap in the prevalence of alcohol use disorder has been rapidly decreasing over time in South Korea. In particular, the prevalence of alcohol use disorder among young women is increasing, ${ }^{41}$ which may be indicative of a phenomenon caused by an increase in women's social advancement and socio-cultural changes, such as the shift to a more permissive drinking culture.

\section{Associations between stress and internet, smartphone, and alcohol use and the mediating effects of resilience and self-esteem}

There were significant correlations between stress and internet use and smartphone use, and these associations were well shown in regression analysis. Stress is thought to play a role in the development of addiction by increasing craving and motivation toward the addictive behavior. ${ }^{42}$ The association between stress and addictive behaviors may be bidirectional; such addictive behaviors may be used as a means of reducing stress, and blunted stress responses may confer an increased risk of engaging in addictive behaviors. ${ }^{42}$

On the other hand, in the regression analysis, there were no association was observed between alcohol and stress as in the previous study, ${ }^{7}$ and this was the same in correlation analysis. Based on studies showing that alcohol is used as a kind of countermeasure to unpleasant things or situations and that alcohol has the effect of self-treatment in buffering stress in life, ${ }^{43}$ it was thought that stress affected alcohol use. However, from a neurobiological point of view, alcohol (substance addiction) directly affects the dopamine system of the brain, whereas behavioral addictions only indirectly affect these systems. ${ }^{44}$ Also, alcohol, unlike behavioral addiction, is accompanied by a physiologically distinct and medically serious withdrawal condition. ${ }^{44}$ In these respects, alcohol could be considered to have a greater effect on addiction. Moreover, alcohol use seems to be more genetically innate than behavioral addictions (e.g., internet use).$^{45}$ Therefore, it could be considered that alcohol causes addiction through a much more complex and diverse pathway that stress alone cannot explain.

Aside from directly affecting addictive behaviors, stress also had indirect effects through resilience and self-esteem in this study. That is, resilience acted as mediating role with respect to the effect of stress on internet use and smartphone use, and self-esteem acted as mediating role with respect to the effect of stress on internet use. The medical students who perceived high stress had lower resilience or self-esteem, resulting in higher levels of addictive behaviors, while those who perceived low stress had higher resilience or self-esteem, contributing to lower levels of addictive behaviors. Resilience and self-esteem were associated with positive emotions, which protect mental health by controlling the negative effects of stress. ${ }^{46,47}$ Moreover, it was found that people with high resilience and people with high self-esteem possess many psychological resources, including tranquility, optimism, and openness, ${ }^{46,48}$ and are more likely to find positive meaning in problems that they have to face. ${ }^{46,48}$ Based on the protective factor model, ${ }^{49}$ these positive personal attributes reduce the negative influence of stress on adaptive behaviors. Thus, people with high resilience and people with high self-esteem are less likely to engage in addictive behaviors.

\section{Implication of mental health for medical students}

According to the results of this study, it would be helpful to provide premedical students with education focused on the primary prevention of addiction. For junior medical students who go through drastic changes in their learning systems, the management of their academic performance and its associated stress is critical. For senior medical students, school authorities need to focus more on the detection and treatment of students at risk because we observed that there were no general increases in addictive behaviors between first and third grade medical students. Moreover, intervention strategies to enhance resilience and self-esteem should be considered, especially with respect to internet and smartphone use, as the improvement of resilience and self-esteem is crucial in alleviating maladaptive coping and in further promoting coping mechanisms ${ }^{48}$ resulting in lower levels of addictive behaviors.

This study has several limitations. First, since our study was a cross-sectional study, we were unable to determine definite causal relations among stress, resilience or self-esteem, and addictive behaviors. Second, the data were obtained through self-reported questionnaires, which could lead to response bias. Third, the ratio of male and female students in this study was approximately 7:3. Although it was proportional to the proportion of the total student distribution, there were a possibility of selection bias. Thus, we have tried to control sex as a covariate in the correlation analysis and mediation analysis. Fourth, due to a lack of data on other variables, including living status, academic performance, and psychological symptoms, the relationships between these factors and addictive 
behaviors were not considered. Fifth, since this study focused on students from a single medical school, we should be careful in generalizing our results. Further prospective studies of medical students of all grades, including those attending other universities, are needed to compensate for our limitations. Nevertheless, our study has the following strengths. For the first time, we investigated the prevalence of various addictive behaviors by grade and their associations with stress through resilience and self-esteem on various addictive behaviors in Korean medical students.

In conclusion, our study suggests that addictions in medical students are as prevalent as among the general population and that the prevalence may increase with increasing grade in medical school. We also suggest that internet use and smartphone use may be better explained by stress than alcohol use, with resilience and self-esteem acting as mediators in their relationships. This study may provide valuable information for the relevant university and medical school authorities responsible for managing addictive behaviors in medical students.

\section{Availability of Data and Material}

The datasets generated or analyzed during the study are available from the corresponding author on reasonable request.

\section{Conflicts of Interest}

Seung Jae Lee, a contributing editor of the Psychiatry Investigation, was not involved in the editorial evaluation or decision to publish this article. All remaining authors have declared no conflicts of interest.

\section{Author Contributions}

Conceptualization: Jimin Lee, Seunghee Won, Seung Jae Lee. Data curation: Jimin Lee, Byung-Soo Kim, Sung Man Chang. Formal analysis: Jimin Lee, Seung Jae Lee. Investigation: Byung-Soo Kim, Sung Man Chang, Seunghee Won. Methodology: Jimin Lee, Seung Jae Lee. Writing_original draft: Jimin Lee, Seung Jae Lee. Writing_-review and editing: Seung Jae Lee, Sung Man Chang. Approval of final manuscript: all authors.

\section{ORCID iDs}

Jimin Lee

Seunghee Won

Sung Man Chang

Byung-Soo Kim

Seung Jae Lee

https://orcid.org/0000-0001-6828-380X

https://orcid.org/0000-0002-4858-6173

https://orcid.org/0000-0001-7077-7171

https://orcid.org/0000-0001-6823-4813

https://orcid.org/0000-0003-3648-9824

\section{Funding Statement}

None

\section{REFERENCES}

1. Pedrelli P, Nyer M, Yeung A, Zulauf C, Wilens T. College students: mental health problems and treatment considerations. Acad Psychiatry 2015; 39:503-511.

2. Kromydas T. Rethinking higher education and its relationship with social inequalities: past knowledge, present state and future potential. Palgrave Commun 2017;3:1-12.

3. Chun KH. Relationship between academic burnout of medical and graduate students and related variables. Korean Med Educ Rev 2014; 16:77-87.
4. Dyrbye LN, Thomas MR, Shanafelt TD. Systematic review of depression, anxiety, and other indicators of psychological distress among US and Canadian medical students. Acad Med 2006;81:354-373.

5. Javaeed A, Zafar MB, Iqbal M, Ghauri SK. Correlation between internet addiction, depression, anxiety and stress among undergraduate medical students in Azad Kashmir. Pak J Med Sci 2019;35:506-509.

6. Lei LY, Ismail MA, Mohammad JA, Yusoff MSB. The relationship of smartphone addiction with psychological distress and neuroticism among university medical students. BMC Psychol 2020;8:97.

7. Frank E, Elon L, Naimi T, Brewer R. Alcohol consumption and alcohol counselling behaviour among US medical students: cohort study. BMJ 2008;337:a2155.

8. Newbury-Birch D, Walshaw D, Kamali F. Drink and drugs: from medical students to doctors. Drug Alcohol Depend 2001;64:265-270.

9. Fahrenkopf AM, Sectish TC, Barger LK, Sharek PJ, Lewin D, Chiang $\mathrm{VW}$, et al. Rates of medication errors among depressed and burnt out residents: prospective cohort study. BMJ 2008;336:488-491.

10. Tsimtsiou Z, Haidich AB, Spachos D, Kokkali S, Bamidis P, Dardavesis $\mathrm{T}$, et al. Internet addiction in Greek medical students: an online survey. Acad Psychiatry 2015;39:300-304.

11. Kim HI, Cheon SH, Kang HJ, Lee K, Jung SP. Associations between smartphone addiction scale and sociopsychological aspects in medical school students. Yeungnam Univ J Med 2017;34:55-61.

12. Alsalameh AM, Harisi MJ, Alduayji MA, Almutham AA, Mahmood FM. Evaluating the relationship between smartphone addiction/overuse and musculoskeletal pain among medical students at Qassim University. J Fam Med Prim Care 2019;8:2953-2959.

13. Shah AA, Bazargan-Hejazi S, Lindstrom RW, Wolf KE. Prevalence of at-risk drinking among a national sample of medical students. Subst Abus 2009;30:141-149.

14. Yoo HH, Cha SW, Lee SY. Patterns of alcohol consumption and drinking motives among Korean medical students. Med Sci Monit 2020;26: e921613.

15. Ministry of Science and Technology Information and Communication. A survey on internet addiction. Available at: https://www.korea.kr/archive/expDocView.do?docId=36557. Accessed August 31, 2021.

16. Park H. Prevalence and related risk factors of problem drinking in Korean adult population. J Kor Acad-Ind Coop Soc 2018;19:389-397.

17. Sinha R. Chronic stress, drug use, and vulnerability to addiction. Ann N Y Acad Sci 2008;1141:105-130.

18. Hassanbeigi A, Askari J, Hassanbeigi D, Pourmovahed Z. The relationship between stress and addiction. Proc Soc Behav Sci 2013;84:13331340 .

19. Younes F, Halawi G, Jabbour H, El Osta N, Karam L, Hajj A, et al. Internet addiction and relationships with insomnia, anxiety, depression, stress and self-esteem in university students: a cross-sectional designed study. PLoS One 2016;11:e0161126.

20. Kim YK. The influence of stress on alcohol addiction, internet addiction and problem gambling: spiritual well-being as mediator. Korean J Couns 2013;14:97-114.

21. Tugade MM, Fredrickson BL. Resilient individuals use positive emotions to bounce back from negative emotional experiences. J Pers Soc Psychol 2004;86:320-333.

22. Robertson TW, Yan Z, Rapoza KA. Is resilience a protective factor of internet addiction? Comput Human Behav 2018;78:255-260.

23. Murphy CM, Stosny S, Morrel TM. Change in self-esteem and physical aggression during treatment for partner violent men. J Fam Violence 2005; 20: 201-210

24. Bahrainian SA, Alizadeh KH, Raeisoon MR, Gorji OH, Khazaee A. Relationship of internet addiction with self-esteem and depression in university students. J Prev Med Hyg 2014;55:86-89.

25. Uba I, Yaacob SN, Talib MA, Mofrad S, Abdullah R. Effect of self-esteem in the relationship between stress and substance abuse among adolescents: a mediation outcome. Int J Soc Sci Human 2013;3:214-217.

26. National Information Society Agency. Third Standardization of Korean 
Internet Addiction Proneness Scale. Seoul: National Information Society Agency; 2011.

27. National Information Society Agency. Development of Korean Smartphone Addiction Proneness Scale for Youth and Adults. Available at: http://library.nia.or.kr/search/detail/CATNAA000000022558. Accessed August 31, 2021.

28. Lee BO, Lee CH, Lee PG, Choi MJ, Namkoong K. Development of Korean version of Alcohol Use Disorders Identification Test (AUDIT-K): its reliability and validity. J Korean Acad Addict Psychiatry 2000;4:8392.

29. Joe KH, Cha SH, Park A, Lee HK, Shin IH, Min SH. Optimum cut-off score for screening of hazardous drinking using the Korean version of Alcohol Use Disorder Identification Test (AUDIT-K). J Korean Acad Addict Psychiatry 2009;13:34-40.

30. Lee J, Shin C, Ko YH, Lim J, Joe SH, Kim S, et al. The reliability and validity studies of the Korean version of the Perceived Stress Scale. Korean J Psychosom Med 2012;20:127-134.

31. Baek HS, Lee KU, Joo EJ, Lee MY, Choi KS. Reliability and validity of the Korean version of the Connor-Davidson Resilience Scale. Psychiatry Investig 2010;7:109-115.

32. Yoo JS, Yang WS, Lee KE, Lee SE, Lee SK, Lee HY, et al. Gender difference in self-esteem of medical students. Korean J Med Educ 2003;15: 241-248.

33. Cohen J. Statistical Power Analysis for the Social Sciences (2nd Ed.). Hillsdale, NJ: Lawrence Erlbaum Associates; 1988.

34. Shankar PR, Balasubramanium R, Ramireddy R, Diamante P, Barton B, Dwivedi N. Stress and coping strategies among premedical and undergraduate basic science medical students in a Caribbean medical school. EIMJ 2014;6:e48-e56.

35. Kardefelt-Winther D. A conceptual and methodological critique of internet addiction research: towards a model of compensatory internet use. Comput Human Behav 2014;31:351-354.

36. Yu S, Sussman S. Does smartphone addiction fall on a continuum of addictive behaviors? Int J Environ Res Public Health 2020;17:422.

37. Erikson EH. Childhood and Society. New York: WW Norton \& Company; 1993.
38. Telzer EH, van Hoorn J, Rogers CR, Do KT. Social influence on positive youth development: a developmental neuroscience perspective. Adv Child Dev Behav 2018;54:215-258.

39. Gunuc S. Peer influence in internet and digital game addicted adolescents: is internet/digital game addiction contagious? Int J High Risk Behav Addict 2016; 6: e33681.

40. Lim K, Meier EB. Different but similar: computer use patterns between young Korean males and females. Educ Technol Res Dev 2011;59:575592.

41. Lee HK. Epidemiology of alcohol use disorders and alcohol policy. J Korean Neuropsychiatr Assoc 2019;58:152-158.

42. McMullin SD, Shields GS, Slavich GM, Buchanan TW. Cumulative lifetime stress exposure predicts greater impulsivity and addictive behaviors. J Health Psychol 2021;26:2921-2936.

43. Colder CR. Life stress, physiological and subjective indexes of negative emotionality, and coping reasons for drinking: is there evidence for a self-medication model of alcohol use? Psychol Addict Behav 2001;15: 237-245.

44. Alavi SS, Ferdosi M, Jannatifard F, Eslami M, Alaghemandan H, Setare M. Behavioral addiction versus substance addiction: correspondence of psychiatric and psychological views. Int J Prev Med 2012;3:290-294.

45. Agrawal A, Verweij KJH, Gillespie NA, Heath AC, Lessov-Schlaggar $\mathrm{CN}$, Martin NG, et al. The genetics of addiction-a translational perspective. Transl Psychiatry 2012;2:e140.

46. Fredrickson BL, Tugade MM, Waugh CE, Larkin GR. What good are positive emotions in crises? A prospective study of resilience and emotions following the terrorist attacks on the United States on September 11th, 2001. J Pers Soc Psychol 2003;84:365-376.

47. Brown J, Marshall M. Self-esteem and emotion: some thoughts about feelings. Pers Soc Psychol Bull 2001;27:575-584.

48. Galanakis MJ, Palaiologou A, Patsi G, Velegraki IM, Darviri C. A literature review on the connection between stress and self-esteem. Psychology 2016;7:687-694.

49. Garmezy N, Masten AS, Tellegen A. The study of stress and competence in children: a building block for developmental psychopathology. Child Dev 1984;55:97-111. 